\title{
Métodos de contracepción en el control poblacional de perros: un punto de vista de los médicos veterinarios de clínica de animales de compañía
}

\author{
Methods of contraception in the population control of dogs: a point of view of veterinarians of pet clinic \\ Mirtha Pelaez ${ }^{1}$, Luisa Echevarría ${ }^{1}$, Diego Soler-Tovar², Néstor Falcón ${ }^{3}$
}

\section{RESUMEN}

El objetivo del estudio fue evaluar los métodos de control de la reproducción de uso potencial en campañas masivas de control poblacional de perros a través de la percepción de médicos veterinarios que laboran en clínica de animales de compañía. En la primera fase del estudio se hizo una revisión de literatura para recopilar información de los métodos de control reproductivos que se encuentran disponibles. En la segunda se definieron los criterios a utilizar para la evaluación de los métodos de control reproductivo (practicidad, económico, seguridad, rapidez y menores efectos secundarios) y la elaboración y validación del instrumento a utilizar en el estudio. En la tercera se aplicó la encuesta a médicos veterinarios $(\mathrm{n}=14)$ que laboraban en el área reproductiva en clínica de animales de compañía. La información fue analizada mediante estadística descriptiva y se estableció un ranking de las metodologías más aceptadas para el control reproductivo de perros. Entre los métodos quirúrgicos, la histerectomía tuvo el mejor puntaje para las características evaluadas. De los métodos químicos el acetato de medroxiprogesterona y el acetato de megestrol en hembras fueron las mejor evaluadas. Los mayores puntajes promedios en general, para los métodos de control de la reproducción de perros, fueron para la orquiectomía $(22,2)$, ovario histerectomía $(20,2)$ y vasectomía $(17,8)$, quienes resultaron como las mejores alternativas según la percepción de los médicos veterinarios evaluadores. La dificultad de sustentar un control poblacional de perros con base a métodos quirúrgicos, hace necesario indagar por otras alterativas; además de exigir cambios en las prácticas y costumbres en el manejo de los perros por parte de los propietarios, en favor de una tenencia responsable de estos animales.

PALABRAS CLAVE: Reproducción, caninos, esterilización, castración.

\section{SUMMARY}

The objective of this study was to evaluate the perceptions of dog birth control methods by using the perceptions of local veterinary clinicians. After a literature review about dog's reproductive control methods the following criteria was established: Simple, affordable, safe, fast and minor side effects. Then, 14 veterinarians were surveyed and the information was analyzed by descriptive statistics resulting in a ranking of the most recommended reproductive control methods. The Hysterectomy had the best score, followed by the pharmacological treatment with Medroxyprogesterone acetate and Megestrol acetate in bitches. For each method the scores were: Orchiectomy

Laboratorio de Reproducción Animal, Facultad de Medicina Veterinaria y Zootecnia, Universidad Peruana Cayetano Heredia. Lima, Perú.

2 Grupo Epidemiología y Salud Pública, Facultad de Ciencias Agropecuarias, Universidad de La Salle. Bogotá, Colombia.

3 Laboratorio de Epidemiología y Salud Pública en Veterinaria, Facultad de Medicina Veterinaria y Zootecnia, Universidad Peruana Cayetano Heredia. Lima, Perú. 
(22.2), Ovary-hysterectomy (20.2) and vasectomy (17.8), all of them were the best alternatives according to the perception of the veterinarians surveyed. In our local context, seems unrealistic the dog population control only by using gonadectomy. We consider necessary to investigate alternative methods involving the participation of the dog owners.

KEYWORDS: Reproduction, canine, gonadectomy, dog population control.

\section{INTRODUCCIÓN}

La tenencia de perros en el Perú está normada por la Ley que Regula el Régimen Jurídico de Canes (Ley 27596) y su reglamento (Decreto Supremo $\mathrm{N}^{\circ}$ 006-2002-SA), normas publicados oficialmente en el año 2001 y 2002, respectivamente (Congreso de la República, 2002; Ministerio de Salud, 2001). Esta normativa busca salvaguardar la integridad, salud y tranquilidad de las personas.

Sin embargo, la tenencia no responsable de perros por parte de los propietarios ha conducido a una reproducción no controlada, fenómeno que se traduce en un aumento de la población de perros vagabundos sin dueño. La Organización Mundial de Sanidad Animal (OIE), considera que estos animales representan un problema de salud pública que requiere ser controlado (Organización Mundial de Sanidad Animal [OIE], 2010). Entre los problemas más importantes asociados a la presencia de perros vagabundos se encuentra la transmisión de enfermedades zoonóticas (rabia, toxocariasis, equinococosis quística, leptospirosis), la contaminación ambiental con heces que presentan huevos o larvas de parásitos (Echinococus granulosus, Toxocara canis) y los accidentes por mordeduras (León, Panta, Yarlequé, y Falcón, 2013).

Una estrategia utilizada para el control de la reproducción de los perros es el desarrollo de campañas de control reproductivo (Escobar, 2008; OIE, 2010), que incluyen la implementación de métodos quirúrgicos y no quirúrgicos.

Entre los métodos quirúrgicos se incluye la esterilización de las hembras basada en ovariohisterectomías (OVH) y las ovariotomías (Muñoz, Vargas, y Soler-Tovar, 2011; Valencia, 2012). La OVH que es la extirpación quirúrgica del útero y los ovarios (Cala, 2014). La técnica de OVH lateral por flanco derecho se popularizó como el método de rutina (Forero, 2006). Por otro lado, la ovariotomía es el nombre que se le da a la extirpación de uno o ambos ovarios (Iglesias, 2002).

En los machos se realiza la orquiectomía y vasectomía (Muñoz et al., 2011; Valencia, 2012). La primera implica el retiro completo de los testículos y en el segundo se exteriorizan los conductos deferentes, los cuales se ligan y se cortan a una distancia de algunos centímetros, suficientes para que no se logre una falsa unión (vasovasostomia) (Lotfe, 1996).

En los métodos no quirúrgicos se incluyen diferentes opciones para hembras y machos; en las hembras se encuentran el control de celos mediante hormonas como progestágenos (acetato de medroxiprogesterona, proligestona, clormadinona y el acetato de megestrol), andrógenos (mibolerona y testosterona), inmunoesterilización y los dispositivos intrauterinos; y en los machos se consideran los anticonceptivos hormonales como progestágenos (acetato de medroxiprogesterona), los análogos de hormona liberadora de gonadotropina GnRH (deslorelina) y la vasectomía química (gluconato de clorhexidina, etanol, formalina y cadmio) (Muñoz et al., 2011; Valencia, 2012).

Ante la necesidad de desarrollar estrategias de control poblacional de perros, el objetivo del estudio fue evaluar los métodos de control reproductivo de uso potencial en campañas masivas de control poblacional de estos a través de la percepción de médicos veterinarios que laboran en clínica de animales de compañía.

\section{MATERIAL Y METODOS}

El estudio se desarrolló en la ciudad de Lima (Perú) y la población objetivo estuvo compuesta por médicos veterinarios colegiados que laboraban en clínicas de animales de compañía. Los participantes fueron elegidos de acuerdo con su especialidad (experticia en el área de reproducción) y años de experiencia (no menor de tres).

El estudio se dividió en tres (3) fases.

En la fase 1 se desarrolló una revisión de literatura como fuente de datos secundarios. Esta etapa buscó obtener la información de interés para el desarrollo de las siguientes fases. La estrategia de búsqueda considero artículos científicos que se encontraban 
en bases de datos reconocidas (Medline a través del buscador de Pubmed, Scopus, LILACS) que ofrecieron información acerca de los métodos de control reproductivos que se encuentran disponibles a escala nacional e internacional e información del uso de los mismos. Al final de esta etapa se obtuvo una matriz considerando el método de control reproductivo y las ventajas y desventajas de los mismos según la literatura.

En la fase 2 se elaboró del instrumento de colección de datos. Para esta fase se convocó a siete (7) médicos veterinarios especialistas en animales de compañía, quienes fueron los encargados de definir los criterios que se utilizaron para la evaluación de los métodos de control reproductivo de los perros y de la elaboración del instrumento que se utilizó en la siguiente fase.

Finalmente, en la fase 3 se aplicó el instrumento elaborado enviándolo a 15 médicos veterinarios. El documento fue enviado por vía electrónica y en forma física con la finalidad de que el profesional tomara la decisión de utilizar cualquiera de ellos para su diligenciamiento. Los instrumentos fueron recogidos después de una semana.

Los criterios de evaluación de los métodos anticonceptivos fueron:

Practicidad: cualidad de un producto de ser práctico de usar, útil y si produce el efecto de contracepción deseado.

Económico: bajo costo en la compra del producto. Seguridad: protección frente a efectos secundarios que pueden ocurrir.

Rapidez: efecto del método reproductivo usado, puede ser inmediato o tardar un tiempo que se encuentra predeterminado en las características del producto.
A partir de la colección de la información, a través del instrumento, se desarrolló una base de datos, que fue analizada mediante estadística descriptiva mediante el paquete estadístico STATA 13.0. Los puntajes asignados (5=Muy Bueno, 4=Bueno, $3=$ Regular, 2=Malo y 1=Muy Malo) a los métodos de control reproductivo de perros por cada criterio de evaluación se sumaron con el fin de establecer un ranking que permitió seleccionar los tres métodos que han de ser consideradas como las mejores. Se utilizaron tablas para mostrar los resultados.

El estudio contó con la aprobación del Comité Institucional de Ética de la Universidad Peruana Cayetano Heredia. La participación de los médicos veterinarios fue voluntaria y la misma quedo consignada mediante la firma de un documento de consentimiento informado.

\section{RESULTADOS}

Se recolectaron 14 encuestas contestadas (de 15 entregadas) con la percepción de médicos veterinarios que trabajaban en clínica de animales de compañía, respecto al uso de métodos anticonceptivos en el control poblacional de perros.

En la evaluación de los métodos quirúrgicos (ovariectomía, ovariohisterectomía, orquiectomía, vasectomía), la histerectomía tuvo el mayor puntaje para las características evaluadas: practicidad, economía, seguridad y menores efectos secundarios (tabla1).

En la evaluación de los métodos químicos, los mayores puntajes se obtuvieron para el acetato de medroxiprogesterona y el acetato de megestrol en hembras (tabla 2). Menores puntajes recibieron los siguientes tratamientos: clormadinona, mibolerona, testosterona, deslorelina, inmunoesterilización,

Tabla1. Evaluación de los métodos quirúrgicos de control poblacional de perros, considerado por médicos veterinarios de práctica clínica en animales de compañía (Lima, Perú).

\begin{tabular}{lccccccccccccc}
\hline \multicolumn{1}{c}{$\begin{array}{c}\text { Características } \\
\text { evaluadas }\end{array}$} & \multicolumn{3}{c}{ Ovariectomía } & \multicolumn{3}{c}{ Ovariohisterectomía } & \multicolumn{3}{c}{ Orquiectomía } & \multicolumn{3}{c}{ Vasectomía } \\
\cline { 2 - 13 }$y$ & $\mathbf{n}$ & Mediana & Moda & $\mathbf{n}$ & Mediana & Moda & $\mathbf{n}$ & Mediana & Moda & n & Mediana & Moda \\
\hline Practicidad & 13 & 3 & 3 & 14 & 5 & 5 & 14 & 5 & 5 & 13 & 3 & 3 \\
Económico & 13 & 3 & 3 & 14 & 3 & 3 & 14 & 3.5 & 3 & 13 & 3 & 3 \\
Seguridad & 13 & 4 & 4 & 14 & 4 & 4 & 14 & 5 & 5 & 13 & 4 & 4 \\
Rapidez & 13 & 4 & 4 & 14 & 3,5 & 3 & 14 & 4,5 & 5 & 13 & 4 & 4 \\
Menor efecto colateral & 11 & 4 & 4 & 12 & 4 & 4 & 12 & 5 & 5 & 11 & 4 & 4 \\
Suma de medianas & - & 18 & - & - & 19,5 & - & - & 23 & - & - & 18 & - \\
\hline
\end{tabular}


Tabla 2. Evaluación de los métodos químicos (progestágenos) para control poblacional de perros considerados por médicos veterinarios de práctica clínica en animales de compañía (Lima, Perú).

\begin{tabular}{|c|c|c|c|c|c|c|c|c|c|c|c|c|}
\hline \multirow{3}{*}{$\begin{array}{c}\text { Características } \\
\text { evaluadas }\end{array}$} & \multicolumn{6}{|c|}{ Acetato de medroxiprogesterona } & \multirow{2}{*}{\multicolumn{3}{|c|}{ Proligestona }} & \multirow{2}{*}{\multicolumn{3}{|c|}{ Acetato de megestrol }} \\
\hline & \multicolumn{3}{|c|}{ En machos } & \multicolumn{3}{|c|}{ En hembras } & & & & & & \\
\hline & $\mathbf{n}$ & Mediana & Moda & n & Mediana & Moda & n & Mediana & Moda & $\mathbf{n}$ & Mediana & Moda \\
\hline Practicidad & 6 & 3,5 & 4 & 10 & 4 & 4 & 5 & 3 & 3 & 6 & 4 & 4 \\
\hline Económico & 6 & 4 & 4 & 10 & 4 & 4 & 5 & 4 & 4 & 6 & 4,5 & 5 \\
\hline Seguridad & 6 & 2,5 & 1 & 10 & 2 & 2 & 5 & 1 & 1 & 6 & 2 & 2 \\
\hline Rapidez & 6 & 3 & 3 & 10 & 3 & 3 & 5 & 3 & 3 & 6 & 3 & 3 \\
\hline $\begin{array}{l}\text { Menor efecto } \\
\text { colateral }\end{array}$ & 5 & 4 & 4 & 10 & 4 & 4 & 5 & 1 & 1 & 6 & 4 & 4 \\
\hline Suma de medianas & - & 17 & - & - & 17 & - & - & 12 & - & - & 17,5 & - \\
\hline
\end{tabular}

Tabla 3. Puntaje medio obtenido en la valoración de los métodos de control poblacional de perros (Lima, Perú).

\begin{tabular}{lccc}
\hline \multirow{2}{*}{ Método } & \multirow{2}{*}{ n } & \multicolumn{2}{c}{ Puntaje } \\
\cline { 3 - 4 } & & Total & Promedio \\
\hline Orquiectomía & 12 & 266 & 22,2 \\
Ovariohisterectomía & 12 & 242 & 20,2 \\
Vasectomía & 11 & 196 & 17,8 \\
Acetato de medroxiprogesterona -machos & 5 & 86 & 17,2 \\
Acetato de medroxiprogesterona- hembras & 10 & 170 & 17,0 \\
Ovariectomía & 11 & 185 & 16,8 \\
Acetato de megestrol & 6 & 98 & 16,3 \\
Proligestona & 5 & 75 & 15,0 \\
\hline
\end{tabular}

dispositivos intrauterinos, gluconato de clorhexidina, etanol, formalina y cadmio.

De acuerdo al resultado promedio calculado para los puntajes otorgados por los médicos veterinarios a los diferentes métodos de control de la reproducción, se encontró que los métodos ovariohisterectomía (20,2 puntos) y orquiectomía (22,2 puntos) fueron las mejores alternativas de control poblacional de perros (tabla 3).

\section{DISCUSIÓN}

El estudio se basó en percepciones de los médicos veterinarios que trabajaban en el área de reproducción dentro de la clínica de animales de compañía. Los resultados muestran que los métodos quirúrgicos (ovario-histerectomía y orquiectomía) son los más valorados para ser utilizados en el control poblacional de perros. Es probable que exista un sesgo, debido a que la experticia de los encuestados se encuentra en este campo, así pues, el uso de otros métodos, como los químicos, se empleó con menor frecuencia. Acuña (2012) indica que en Estados Unidos, la técnica preferida es la ovario-histerectomía, pero en países como Argentina se ha implementado la ovariectomía con buenos resultados.

Valencia-Ruiz, Diaz-Bravo, y Garcia-Duran (2012) hablan de los beneficios de la esterilización; menciona que en hembras se reduce o elimina la incidencia de piometra y tumores mamarios; en machos se reduce la posibilidad de desarrollar patologías prostáticas como neoplasias, además se reduce el comportamiento agresivo y la posibilidad de que éstos escapen y deambulen por la vía pública donde pueden atacar a otras personas o animales, ser atacados ellos mismos o sufrir accidentes. La Sociedad Mundial para la Protección Animal, Sociedad Humanitaria Internacional, Fondo Internacional para el Bienestar Animal, y la RSPCA Internacional (2010) recopilan información de diversos estudios que indican que la 
esterilización temprana es más efectiva en evitar el nacimiento de camadas accidentales. Además, es posible esterilizar con seguridad cachorros desde 6-8 semanas de edad sin efectos negativos a largo plazo.

Pérez (2009) indica que los procedimientos quirúrgicos en hembras (ovarihisterectomía) y en machos (orquiectomía) eliminan de manera definitiva la función reproductiva de los perros. En tanto Kiener et al. (2013), menciona que los métodos quirúrgicos son los más eficientes en el control de la reproducción, señalando que en Francia se observó que el mayor número de castraciones eran en hembras y que los métodos no quirúrgicos no tenían mucha acogida entre los pobladores de la zona.

Según Ruiz (2011), los animales hembras son generalmente destinadas a esterilizaciones quirúrgicas en comparación a los machos, lo que se podría atribuir a factores culturales. Sin embargo, el costo de las esterilizaciones en hembras es mayor que la de machos y esta puede ser una limitante en los programas de control poblacional de perros en zonas vulnerables, debido a las condiciones sociales, culturales y económicas de estas poblaciones. Además, la esterilización en hembras requiere de ambientes con condiciones mínimas de asepsia, por lo que son discutibles las campañas de control masivo de la reproducción promovidas por algunas organizaciones proteccionistas $\mathrm{u}$ otras instituciones privadas $\mathrm{y}$ públicas.

Acuña (2012) indica que la ovariohisterectomía está contraindicada en distensiones uterinas por gestación, piometra, edad del animal u obesidad; además de indicar que la incidencia de complicaciones varían entre un 20 a $26 \%$ de los casos, predominando complicaciones de la herida quirúrgica y las hemorragias.

Una campaña de control poblacional de perros debe estar basada en el conocimiento de dicha población y sus características demográficas (edad, sexo, tamaño, raza, entre otras). León, Soriano, Arauco, y Falcón (2014), mencionan que el desconocimiento del tamaño de la población de perros no permite presupuestar, ni programar actividades de control poblacional. Por ello, es importante y necesario que las autoridades de salud y de los gobiernos locales se preocupen por obtener información actualizada acerca de la población de perros con dueños y de libre intinerancia dentro de sus respectivas localidades, a través de estudios de estimación de población de perros o censos. Amaku,
Dias, y Ferreira (2009) mencionan que, en la ciudad de Sao Paulo, la esterilización no logró los resultados deseados debido a la baja tasa de esterilización, por no saber el número de perros que se tenía y debía castrar.

Mackie (2012) indica que la esterilización debe de alcanzar una cobertura mínima del $70 \%$ de la población de perros, a fin de que se observe una reducción en la población de los mismos. Sin embargo, Amaku et al. (2009) mencionam que en la ciudad de Sao Paulo, incluso a altas tasas de esterilización (por ejemplo, 0,80 al año), se necesitarían alrededor de 5 años de campañas para permitir una reducción del $20 \%$ en la densidad de la población.

La mayoría de médicos veterinarios no ofrecieron más información acerca de los métodos no quirúrgicos, argumentando que no son utilizados con frecuencia, por lo tanto, tampoco son sugeridos a los propietarios de perros. Respecto al uso de sustancias hormonales, Flores (2006) menciona que al usar estrógenos, se pueden producir efectos secundarios severos como: trombocitopenia, leucopenia, anemia, hiperplasia quística endometrial, hemorragias internas, depresión de la médula ósea y, debido a esto, se puede causar la muerte en algunos casos, quistes ováricos, piometra y endometritis. Ayala, Chahín, y Jovel (2014) señalan que un producto a base de péptido sintético análogo de la GnRH con toxoide diftérico, se aplica directamente sobre la cola del epidídimo de los perros machos, lo que trae como consecuencia la paralización inmediata de los espermatozoides con los que tiene contacto el compuesto.

Por otro lado, en caso de la inmunoesterilización, Flores (2006) indica que estos son anticuerpos que bloquean la fertilización primaria en el sitio de interacción del espermatozoide con la zona pelúcida (alteran el acoplamiento entre el espermatozoide y la zona pelúcida o modifican la habilidad del espermatozoide a penetrar a la misma).

En un entorno en donde la tenencia de perros se desarrolla sin mayor responsabilidad por parte de sus dueños, el control de la reproducción de los perros es importante, a fin de evitar el impacto de esta población sobre la salud pública. Sin embargo, Zumpano, Tortosa, y Degregorio (2011) mencionan que para que haya un adecuado control de las poblaciones de perros, se requieren cambios en las comportamientos de la población humana y así no tener más animales abandonados y tener menor cantidad de animales vagabundos con dueño o sin dueño. Por ello, el control 
de la población de perros no solo se ha de lograr con la aplicación de métodos de control reproductivo quirúrgico o químico; se requiere también cambiar la cultura y las prácticas de la población hacia una tenencia responsable de los animales de compañía.

En este sentido, las autoridades educativas tienen una gran responsabilidad en la sensibilización de la población, a fin de convertirlos en un aliado en la lucha contra esta problemática. La educación en tenencia responsable de los animales de compañía, además, evitará la exposición a enfermedades zoonóticas, la contaminación ambiental y los accidentes por mordeduras. A ello, debe sumarse, como último recurso, la eutanasia, que debe usarse en zonas donde el esfuerzo por reubicar a los perros sin dueños fracase.

\section{Correspondencia:}

Mirtha Pelaez Rueda

Correo electrónico: mirtha.pelaez@upch.pe

\section{REFERENCIAS BIBLIOGRAFICAS}

1. Acuña. G. (2012). Complicaciones intraoperatorias y postoperatorias de ovario-histerectomía por flanco en perras, utilizada en programas públicos de control de natalidad en Chile. (Tesis de médico veterinario, Universidad de Chile, Santiago, Chile).

2. Amaku, M., Dias, R., \& Ferreira, F. (2009). Dinâmica populacional canina: potenciais efeitos de campanhas de esterilização. Rev Panam Salud Pública, 25(4), 300-4.

3. Ayala, W., Chahín, C., \& Jovel, E. (2014). Evaluación del péptido sintético análogo del GnRH con toxoide diftérico, como método contraceptivo en Canis lupus familiaris machos en el cantón primavera, municipio de Santa Ana, departamento de Santa Ana, El Salvador. (Tesis de Médico Veterinario Zootecnista, Universidad del Salvador, San Salvador, El Salvador).

4. Cala, F. (2014). TÉCNICA LATERAL Ovariohisterectomía (OVH) lateral. REDVET, 15(2), 1-12.

5. Congreso de la República. (14 de diciembre del 2001). Ley $N^{\circ} 27596$ - Ley que regula el Régimen Jurídico de Canes. Lima, Perú: Diario Oficial El Peruano.

6. Escobar, L. (2008). Evaluación de comportamiento de perros machos: aprendizaje hacia el control de poblaciones caninas. Puerto Natales, Chile: Servicio Agrícola y Ganadero, Gobierno de Chile.

7. Flores, V. (2006). Esterilización canina y felina con la finalidad de controlar el problema de la rabia en el Municipio de Naucalpan de Juárez Estado de
México. (Tesis de Médico Veterinario Zootecnista, Universidad Nacional Autónoma de México, Juárez, Mexico).

8. Forero, G. (2006). Ovariohisterectomía (OVH), técnica lateral. REDVET, 7(6), 0-0. Recuperado de: http://www.veterinaria.org/revistas/redvet/ n060606/060608.pdf

9. Sociedad Mundial para la Protección Animal, Sociedad Humanitaria Internacional, Fondo Internacional para el Bienestar Animal, \& RSPCA Internacional. (2010). GUÍA PARA EL MANEJO HUMANITARIO DE POBLACIONES CANINAS Coalición Internacional para el Manejo de Animales de Compañia. Washington, USA: FAO.

10. Iglesias, J. (2002). La ovariotomía: Una breve reseña del inicio de la cirugía abdominal. Med Univer, 4(17), 249-55.

11. Kiener, M., Fusari, M., Serafino, L., Barolin, J., Dolzani, M., Sturtz, W.,...Romano, G. (2013). Métodos anticonceptivos utilizados en la población de mascotas de La Francia. XIV Jornadas de Divulgación Técnico-Científicas 2013, Jornada Latinoamericana. Facultad de Ciencias Veterinarias, Universidad Nacional de Rosario. Bogotá, Colombia.

12. León, D., Panta, S., Yarlequé, C., \& Falcón, N. (2013). La convivencia con mascotas en zonas periurbanas: experiencia en Lima - Perú. Revista de ciencias veterinarias, 29(4),21-25.

13. León, D., Soriano, J., Arauco, D., \& Falcón, N. (2014). Estimación de la población de perros con dueño y perros vagabundos: Importancia para la salud pública. MV Rev de Cien, 30(3), 5- 10.

14. Lotfe, A. (1996). Evaluación de la técnica modificada de vasectomía por ligadura y extirpación del conducto deferente en caninos domésticos. (Tesis profesional Médico Veterinario y Zootecnista, Universidad de Guadalajara, Las Agujas, Nextipac, Zapopan, Jalisco, Mexico).

15. Mackie, M. (2012). La Regla del 70\%. 1ra Conferencia Internacional de Esterilización de Perros y Gatos. CEFUTREMA, Puebla, México.

16. Ministerio de Salud (21 de junio del 2002). Reglamento de la ley $N^{\circ} 27596$ que regula el régimen jurídico de canes. Lima, Perú: Diario Oficial El Peruano.

17. Muñoz, M., Vargas, I., \& Soler-Tovar, D. (2011). Métodos para el control de población canina: introducción. Sapuvet, 2(1),63-79.

18. Organización Mundial de Sanidad Animal. (2010). El control de las poblaciones de perros vagabundos.

19. En Organización Mundial de Sanidad Animal. Código Sanitario para los Animales Terrestres. Paris, Francia: Organización Mundial de Sanidad Animal.

20. Pérez, M. (2009). La sobrepoblación de perros no domiciliados: un problema social vinculado con la difícil tarea de educar. Temas de Ciencia y Tecnología, 
37(4),45-48. Recuperado de: http://www.utm.mx/ edi_anteriores/temas037/N4.pdf

21. Ruiz, A. (2011). Nivel de conocimiento de los propietarios sobre aspectos reproductivos del perro (Canis lupus familiaris) en dos grupos socioeconómicos de la ciudad de Valdivia, Chile. (Tesis de médico veterinario, Universidad Austral de Chile, Valdivia, Chile).

22. Valencia, C. (2012). Técnicas de control de poblaciones caninas callejeras usadas a nivel mundial. (Tesis de médico veterinario, Universidad Austral de Chile, Valdivia, Chile).
23. Valencia-Ruiz, M., Diaz-Bravo, L., \& Garcia-Duran, R., (2012). Descripción y usos del método Delphi en investigaciones del área de la salud. Inv Ed Med, 1(2), 90-95.

24. Zumpano, R., Tortosa, A., \& Degregorio, O. (2011). Estimación del impacto de la esterilización en el índice de crecimiento de la población de caninos. Rev Inv Vet Perú, 22 (4), 336-341. 